\title{
Human Transcriptome and Chromatin Modifications: An ENCODE Perspective
}

\author{
Li Shen ${ }^{1}$, Inchan Choi ${ }^{2}$, Eric J. Nestler ${ }^{1}$, Kyoung-Jae Won ${ }^{2 *}$ \\ ${ }^{1}$ Department of Neuroscience, Mount Sinai School of Medicine, New York, NY 10029, USA, \\ ${ }^{2}$ Department of Genetics, Institute for Diabetes, Obesity and Metabolism, \\ University of Pennsylvania, Philadelphia, PA 19104, USA
}

\begin{abstract}
A decade-long project, led by several international research groups, called the Encyclopedia of DNA Elements (ENCODE), recently released an unprecedented amount of data. The ambitious project covers transcriptome, cistrome, epigenome, and interactome data from more than 1,600 sets of experiments in human. To make use of this valuable resource, it is important to understand the information it represents and the techniques that were used to generate these data. In this review, we introduce the data that ENCODE generated, summarize the observations from the data analysis, and revisit a computational approach that ENCODE used to predict gene expression, with a focus on the human transcriptome and its association with chromatin modifications.
\end{abstract}

Keywords: chromatin modification, ENCODE, GENCODE, transcriptome

\section{Introduction}

In September 2012, 30 research papers, including 6 in Nature, were published online (http://www.nature.com/ encode/) about genomescale data from a decade-long project, the Encyclopedia of DNA Elements (ENCODE) [1]. Aiming to delineate all functional elements encoded in the human genome [1-4], the ENCODE project examined $1 \%$ of the human genome in its pilot phase and scaled up to the whole genome in its second phase. It released data from more than 1,600 sets of experiments from 147 types of tissues [4]. These data include a catalog of human proteincoding and noncoding RNAs as well as protein-DNA interactions, chromatin and DNA accessibility, histone modifications, DNA methylation, and long-range chromosomal interactions. The ENCODE consortium reported that $80.4 \%$ of the human genome serves some type of known biochemical function [4]. The unprecedented volume and span of this study will make it an excellent resource for biological analyses. On the other hand, it can be overwhelming for a researcher to access and interpret the data. ENCODE also developed novel (or refined existing) techniques for data generation and computational analyses. These techniques are by no means restricted to ENCODE and can be employed in a wide array of applications. Because of the vast span of ENCODE, we will limit our scope to the human transcriptome and its association with chromatin modifications in this review. We aim to introduce the data that ENCODE generated and the techniques (both experimental and computational) that were used to generate them. We summarize the observations that ENCODE found. We also provide a detailed discussion of a machine learning method that was used to predict gene expression from chromatin modifications with higher accuracy than its predecessors.

\section{Chromatin Modifications Measured by Chromatin Immunoprecipitation Sequencing (ChIP-seq)}

DNA sequences are wrapped around octamers of histone proteins to form nucleosomes, the unit of chromatin. The nucleosome core is composed of two copies each of four histone proteins. Each histone has an N-terminal tail that faces outward from the nucleosome and can be chemically modified to influence the accessibility of the chromatin and interactions with other chromatin-binding proteins. These 
histone modifications are associated with the activation or repression of gene transcription [5] and many other activities of the genome, such as enhancer activities [6] and splicing regulation $[7,8]$. There has been great interest in dissecting the interactions between histones and other chromatin modifications and transcriptional regulation in recent years [9]. One of ENCODE's major goals [4] is to use these chromatin modifications to define regulatory elements of the human genome in multiple cell lines and to investigate their interactions with RNA transcription.

Chromatin immunoprecipitation (ChIP) is an experimental technique used to investigate protein-DNA interactions in the cell. ChIP, followed by microarray (ChIP-chip) or high-throughput sequencing (ChIP-seq), is widely used to determine genomewide binding locations of proteins, including transcription factors, covalent modifications of histones, and other chromatin regulatory proteins. ChIPchip was the chosen technology for the pilot phase of ENCODE to dissect the regulatory regions in the $1 \%$ selected portion of the human genome $[2,10,11]$. As nextgeneration sequencing technology advanced [12], it soon became clear that ChIP-seq was a superior approach $[13,14]$.

ChIP-seq was the chosen technology for the second phase of the ENCODE project $[3,4,15]$. Although ChIP-seq shows clear advantages over ChIP-chip, it is by no means a perfect technology. In an evaluation study performed by Chen et al. [16], multiple factors were found to influence the fidelity of ChIP-seq data. For example, ChIP-seq data were found to be biased toward open chromatin regions, leading to false positives if not corrected; comparison of different algorithms also showed notable variation in sensitivity and specificity.
The ENCODE consortium has established a set of guidelines to ensure the quality of ChIP-seq experiments [15]. Because antibodies play a predominant role in the success of ChIP experiments, a significant effort has been made by ENCODE to characterize the specificity and efficiency of a large number of antibodies. The list of the antibodies used and validated by ENCODE can be found at: http://genome. ucsc.edu/ENCODE/antibodies.html. A large-scale assessment of histone modification antibodies $(>200)$ was performed by multiple groups [17] of the ENCODE consortium, with the most up-to-date information available at: http://compbio.med.harvard.edu/antibodies/. In addition, multiple quality metrics have been developed and used by the ENCODE project [15], as listed in Table 1. Having employed these quality metrics, the ENCODE consortium mapped 11 histone modifications plus one histone variant across 46 human cell types, including a complete matrix of the 12 marks across two groups of cell lines, designated as tier 1 and tier 2 .

\section{Transcriptome in Human Cells \\ ENCODE releases a reference gene set (GENCODE) and RNA expression catalogs}

The ENCODE project has produced a reference gene set, referred to as GENCODE (http://www.gencodegenes.org) [18]. GENCODE (version 7) identified a comprehensive set of 20,687 protein-coding and 9,640 manually curated long noncoding RNA (lncRNA) loci (representing 15,512 noncoding transcripts/isoforms). Currently, in version 15 , it has 20,447 coding genes and 13,249 lncRNA loci (representing

Table 1. Quality metrics of chromatin modification ChIP-seq data employed by ENCODE

\begin{tabular}{|c|c|c|c|}
\hline Name & Definition & Measures & Requirements \\
\hline Sequencing depth & The number of uniquely mapped reads & Sufficiency of sequencing & $\begin{array}{l}>10 \text { million for sharp peak } \\
>20 \text { million for broad peak }\end{array}$ \\
\hline NRF & $\mathrm{NRF}=\frac{\text { \#uniquely mapped locations }}{\text { \#uniquely mapped reads }}$ & PCR bottlenecking & $\mathrm{NRF} \geq 0.8$ \\
\hline FRiP & $\mathrm{FRiP}=\frac{\text { \#mapped reads in peaks }}{\text { \#total mapped reads }}$ & Signal-to-noise ratio & $\mathrm{FRiP}>1 \%$ \\
\hline CC & $\begin{aligned} \mathrm{NSC} & =\frac{\mathrm{CC} \text { (fragment lenth) }}{\min (\mathrm{CC})} \\
\mathrm{RSC} & =\frac{\mathrm{CC} \text { (fragment lenth) }-\min (\mathrm{CC})}{\mathrm{CC} \text { (phantom peak) }-\min (\mathrm{CC})}\end{aligned}$ & ChIP enrichment & $\begin{array}{l}\mathrm{NSC} \geq 1.05 \\
\mathrm{RSC} \geq 0.8\end{array}$ \\
\hline IDR & $\begin{array}{l}\text { The posterior probability for a peak being in } \\
\text { the irreproducible group }\end{array}$ & $\begin{array}{l}\text { Reproducibility between } \\
\text { replicates }\end{array}$ & IDR $<0.01$ \\
\hline $\begin{array}{l}\text { Annotation } \\
\text { enrichment }\end{array}$ & $\begin{array}{l}\text { Enrichment of chromatin-associated modifications and } \\
\text { proteins at functionally annotated features, } \\
\text { such as TSS and TES }\end{array}$ & $\begin{array}{l}\text { Known characteristic } \\
\text { enrichment }\end{array}$ & $\begin{array}{l}\text { Not specified; based on } \\
\text { human experts }\end{array}$ \\
\hline
\end{tabular}

ChIP-seq, chromatin immunoprecipitation sequencing; ENCODE, Encyclopedia of DNA Elements; NRF, nonredundancy fraction; PCR, polymerase chain reaction; FRiP, fraction of reads in peaks; CC, cross correlation; NSC, normalized strand cross-correlation; RSC, relative strand cross-correlation; IDR, irreproducible discovery rate; TSS, transcription start site; TES, transcription end site. 
Table 2. Comparison of IncRNAs and coding RNAs

\begin{tabular}{|c|c|c|}
\hline Property & IncRNAs & Coding RNAs \\
\hline Protein coding potential & Very weak & Higher \\
\hline Structure & $\begin{array}{l}\text { Majority has } 2 \text { exons ( } 42 \%) \\
\text { Longer exons and introns }\end{array}$ & $\begin{array}{l}6 \% \text { has } 2 \text { exons, } 75 \% \text { has at least two } \\
\text { different/dominant major isoform }\end{array}$ \\
\hline Evolutionary conservation & $\begin{array}{l}\text { Weaker } \\
30 \% \text { primate specific }\end{array}$ & Stronger \\
\hline Chromatin marks & $\begin{array}{l}\text { Active histone marks at TSS } \\
\text { Slight excess level of silencing (H3K27me3) and } \\
\text { activating (H3K36me3) marks }\end{array}$ & Active histone marks at TSS \\
\hline Class & $\begin{array}{l}\text { Predominantly in nucleus } \\
\text { Significantly more enriched in chromatin than mRNAs }\end{array}$ & Predominantly in cytosol \\
\hline
\end{tabular}

LncRNA, long noncoding RNA; TSS, transcriptional start site.

22,531 noncoding transcripts/isoforms). For this reference gene set, GENCODE used manual gene annotation from the Human and Vertebrate Analysis and Annotation (HAVANA) group (http://www.sanger.ac.uk/research/projects/verteb rategenome/havana/) and automatic gene annotation from Ensembl [19]. To construct a comprehensive RNA expression catalog, ENCODE sequenced RNA (using RNAseq) from 15 different cell lines in multiple subcellular fractions [18]. The expression catalog in multiple cell types provides the transcriptome of coding and noncoding genes, short ( $<200 \mathrm{bp}$ ) and long (>200 bp) in length, polyadenylated or nonpolyadenylated, as well as the compartment in cells where RNAs in each type are populated.

\section{Long noncoding RNAs}

LncRNAs are nonprotein-coding transcripts longer than 200 nucleotides. LncRNAs have been known to have similar characteristics as coding RNAs. They are polyadenylated, associated with chromatin signatures, and have multiexonic structure [20, 21]. Some lncRNAs use identical or almost identical transcription initiation complexes [22] and sometimes overlap with protein-coding genes and can be transcribed from either strand $[22,23]$.

GENECODE interrogated the properties of 15,512 lncRNAs. Table 2 summarizes the comparison between lncRNAs and coding RNAs. Expressed lncRNAs have an activating histone modification profile similar to that of protein-coding genes, with slightly excess levels of both silencing (H3K27me3) and activating (H3K36me3) marks in lncRNAs [24]. Although many lncRNAs are polyadenylated, they are significantly enriched in nonpolyadenylated transcripts compared with coding RNAs [24]. As expected, the lncRNAs have significantly lower protein potentials compared with mRNAs. Interestingly, they are biased toward two-exon transcripts and predominately localized in the chromatin and nucleus, while coding RNAs are predominantly observed in the cytosol; only $6 \%$ of them have a 2-exon structure.

In order to identify the function and targets of lncRNAs, Derrien et al. [24] investigated the correlation between lncRNAs and coding RNAs; both positive and negative correlations were found. Overall, the number of positive correlations was larger than the negative correlations. Compared with trans-acting lncRNAs, cis-acting lncRNAs showed more positive correlations. Interestingly, lncRNAs that intersected protein-coding exons in the antisense orientation showed a strong pattern of coexpression [24]. They also checked sequence conservation by BLASTing human IncRNAs against all available mammalian genomes. Around $30 \%(n=4,546)$ of $1 n c R N A s$ appeared to have arisen in the primate lineage, and $0.7 \%(n=101)$ of them appeared to be human-specific [24].

\section{RNA splicing}

Recent studies suggest that many pre-mRNA processing events are cotranscriptional [25-27]. RNA imaging found that splicing can follow the completion of transcription [28]. Tilgner et al. [29] investigated cotranscriptional splicing by interrogating RNA fractions from several cellular compartments in K562 cells. They found that only a tiny fraction of exons were found to be surrounded completely by an unspliced intron in chromatin-associated RNA. This suggests that splicing is already occurring during transcription [18, 29]. The strong enrichment of spliceosomal small nuclear RNAs (snRNAs) in the chromatin-associated fraction compared with other fractions also supports cotranscriptional splicing. These observations confirm the idea that chromatin structure could play a role in splicing [7, 30-34]. However, for alternative exons and lncRNAs, splicing tends to occur later and might remain unspliced in some cases [29].

\section{RNA editing}

Li et al. [35] reported 10,210 exonic sites in the human 
genome where an RNA sequence did not match with the DNA sequence, suggestive of RNA editing. However, there have been debates about whether their observations occurred by sequencing error, gene duplication, mapping error, or read-end misalignment [36-38].

Park et al. [39] developed a pipeline to filter sequencing artifacts in identifying RNA editing. They found that the majority of non-A-to-G variants came from incorrect read mapping across splice junctions. Most of the edits they found were A-to-G(I) variants, which corresponds with recent observations [40] but differs from Li et al.'s report [35] of a substantial number of noncanonical single nucleotide variant edits in the RNA of human lymphoblastoid cells. Most A-to-G(I) edits were located in introns and untranslated regions, with only a fraction of sites reproducibly edited across multiple cell lines.

\section{Pseudogenes}

Pseudogenes have been considered nonfunctional sequences of genomic DNA that lost their coding potential due to disruptive mutations, such as frameshifts and premature stop codons [41-44]. Recent studies have shown that pseudogenes can regulate their parent genes [45-49]. Using manual annotation, with the assistance of computational pipelines, GENCODE created a database called Pseudogene Decoration Resource (psiDR). psiDR provides a variety of information on 11,216 peudogenes, including transcription activity, chromatin features, functional genomics, and evolutionary constraints [50]. Transcribed pseudogenes show enhanced chromatin accessibility and enrichment with histone marks, although they are lower than those of coding genes. The majority of pseudogenes contains no or very few transcription factor binding sites (TFBSs), but the diffe- rences between the number of TFBSs associated with transcribed and nontranscribed pseudogenes are significant.

\section{Small RNAs}

GENCODE also categorized 7,054 small RNAs into 2,756 micro RNAs (miRNAs), snRNAs, small nucleolar RNAs (snoRNAs), and transfer RNAs (tRNAs). They found that miRNAs and tRNAs were abundant in cytosol, snoRNAs were in the nucleus, and snRNAs were in both the nucleus and cytosol. snRNAs were found abundantly in the chromatin-associated RNA fractions, which further supports predominant splicing during transcription.

\section{Enhancer RNAs}

RNAs at enhancers (eRNAs) were first characterized by observing transcription activities at the promoter-distal CREB binding protein-binding sites in mouse cortical neurons [51]. The bidirectional property of eRNAs and their association with gene expression were further studied using nascent RNAs from global run-on sequencing (GROseq) data $[52,53]$. ENCODE used RNA assays to detect transcription activity. Besides the bidirectional property, ENCODE identified transcriptional initiation using cap analysis gene expression (CAGE) [54] signals. Interestingly, they found polyadenylated eRNAs, although most eRNAs were prevalent in the nonpolyadenylated form. They also observed that histone marks associated with eRNAs were the factors for transcriptional initiation and elongation: H3K27ac, H3K79me2, and RNA polymerase. These lines of evidence suggest regulatory functions of eRNAs.

Table 3. Summary of data collected for modeling gene expression using chromatin features

\begin{tabular}{|c|c|c|}
\hline Description & Classification & Detail \\
\hline \multirow[t]{3}{*}{ Chromatin } & Methylation & $\begin{array}{l}\text { H3K4me1, H3K4me2, H3K4me3, H3K27me3, H3K36me3, H3K79me2, H3K9me1, } \\
\text { H3K9me3, H4K20me1 }\end{array}$ \\
\hline & Acetylation & H3K9ac, H3K27ac \\
\hline & Others & H2A.Z, DNase I hypersensitivity \\
\hline \multirow[t]{2}{*}{ Cell lines } & Tier 1 & K562, GM12878, H1-hESC \\
\hline & Tier 2 & HepG2, HeLa-S3, NHEK, HUVEC \\
\hline \multirow[t]{3}{*}{ Cellular compartments } & Nucleus & Further isolated into nucleolus, nucleoplasm, and chromatin in selected cell lines \\
\hline & Cytosol & \\
\hline & Whole cell & \\
\hline \multirow{2}{*}{ RNA extractions } & PolyA + & \\
\hline & PolyA- & \\
\hline \multirow[t]{3}{*}{ RNA sequencing technology } & CAGE & Cap analysis of gene expression [54] \\
\hline & RNA-PET & RNA paired end tag [55] \\
\hline & RNA-seq & Whole transcript coverage [56] \\
\hline
\end{tabular}




\section{Predicting Gene Expression by Chromatin Features}

Enabled by the unprecedented volume of data generated by the ENCODE project, Dong et al. [57] performed a very interesting study that attempted to predict gene expression from chromatin features using machine learning techniques. As usually practiced, a machine learning study is composed of a series of procedures that typically involve data collection, data representation, model building, and testing. Using machine learning terminology, the response variables here are gene expression patterns that are predicted/modeled, while the predictors or features are various chromatin measures. Chromatin data were collected from the ENCODE project with 11 chromatin modifications, one histone variant, and DNase I hypersensitivity, all mapped in seven cell lines (Table 3). Gene expression data were from different cellular compartments, using two different RNA isolation approaches and sequenced with different technologies (Table 3). With such diversity of RNA sources, Dong et al. [57] answered not only the general question of whether gene expression can be predicted with satisfactory accuracy but also the questions of whether different RNA sources that are sequenced by different technologies can be predicted differently using the same chromatin features.

The gene expression data are relatively easy to represent. They were separated into two classes: transcriptional start site (TSS)-based and transcript-based (Tx-based). TSSbased expression data are read counts within a 101-bp window centered on the TSS, which measures transcriptional initiation. Tx-based expression data are summarized read counts from the whole transcript, which measures transcriptional elongation. However, the representation of chromatin data seems to be tricky and requires further research. Dong et al. [57] used a strategy called "bestbin," which considers the chromatin signals across the entire gene body, including 2-kb flanking regions. It basically segregates each genic region into equal bins of $100 \mathrm{bp}$ and summarizes the chromatin signals within each. A training dataset was used to identify the bin that correlates most with gene expression, and the learned parameter values were applied to testing data. Other strategies $[58,59]$ are possible, but the "bestbin" strategy was found to be superior [57].

RNA sequencing data are known to contain very little or no background noise, with a large portion of the genes having 0 read counts. Therefore, the response variables become a mixture of a 0 component and a positive counting component. Neither a classifier nor regression method seems to be able to capture the variability of both. To deal with the challenge, a two-step approach was used by [57], so that a classifier first categorized genes as "expressed" or "unexpressed," Then, a regression method was used to predict the expression levels of the expressed genes. The final prediction is the product of the classifier and the regression method.

To test the performance of their approach, each dataset was separated into a training set and a testing set. On the training set, the "best bin" and a few other parameters were determined. After that, a 10-fold crossvalidation was performed on the testing set to evaluate the model. AUC [60] was used to represent the accuracy of the classifier. Two criteria were used to represent the accuracy of the regression method. Pearson correlation coefficient (PCC) was used to measure the similarity between the predicted value and experimental value. Root mean square error was used to measure the disparity between the predicted value and experimental value.

Overall, the two-step model achieved very satisfactory performance, with a PCC $>0.9$ for a number of datasets and a PCC $>0.8$ for $71 \%$ of the whole data. Looking at the two steps separately, the AUC can be as high as 0.95 for the classifier, and the PCC can be as high as 0.77 for the regression method when predicting CAGE-measured polyA+ cytosolic RNA expression in K562 cells. Similar performance was achieved in other datasets. It was also found that $\mathrm{H} 3 \mathrm{~K} 9 \mathrm{ac}$ and $\mathrm{H} 3 \mathrm{~K} 4 \mathrm{me} 3$ are the most important predictors for the classifier, strengthening their roles as activation marks at TSSs. In contrast, H3K79me2 and H3K36me3 are the most important predictors for the regression methods, strengthening their roles as elongation marks at gene bodies. These findings show that the two-step model not only improved the accuracy of prediction but also enabled the identification of the chromatin features that are associated with different transcriptional roles.

\section{Discussion}

During the past decade, the ENCODE project has evolved into a genomewide scale, and the dataset it generated has expanded in quantity as well as in scope. The ENCODE project has provided a global view about the human transcriptome and most noticeably found that the transcribed region of the human genome is more abundant than we previously thought. This finding significantly reduced the so-called intergenic regions, as defined in the traditional sense. The quantitative measurement of RNA species in several cellular compartments as well as their polyadenylation provided a comprehensive view of RNA generation. In this review, we have revisited the characteristics of both coding and noncoding transcripts in association with their structures and locations in cells. 
Besides the human transcriptome and the associated chromatin modification data that we discussed, the ENCODE consortium also mapped transcription factor binding sites and their associated DNA motifs, as well as DNA methylation and long-range chromosomal interactions [4]. In parallel, the Roadmap Epigenomics Project (http:// www.roadmapepigenomics.org) and International Human Epigenome Consortium (http://www.ihec-epigenomes.org) have been accumulating data of a similar scale to understand the human genome in other tissues and conditions. It is remarkable that the ENCODE data altogether have associated more than $80 \%$ of the human genome with some type of biochemical function so far, and the coverage will continue to increase as we map additional protein-DNA interactions in the near future. It has now become very clear that so-called "junk DNA" is not evolutionarily vestigial but has specific structural or biochemical functions.

While data generation has been a major goal of ENCODE, the need to integrate the current datasets is becoming more and more important. Computational approaches have been developed to exploit the ENCODE data at to a fuller potential. For instance, chromatin features were used to model gene expression $[57,61]$; integrative methods were developed to annotate genomes [62-64]; visualization tools were developed to investigate epigenomic regulation at a global scale (also see ngs.plot at https://code.google. $\mathrm{com} / \mathrm{p} /$ ngsplot/) $[65,66]$; and large regulatory networks were reconstructed, based on TFs and DNaseI footprinting $[67,68]$. The network-based approach as well as the chromosomal interactions [69] provided novel angles in studying gene regulation at higher levels. New approaches to integrate the large amount of data to provide new biological insights are on the horizon.

\section{References}

1. ENCODE Project Consortium. The ENCODE (ENCyclopedia Of DNA Elements) Project. Science 2004;306:636-640.

2. ENCODE Project Consortium, Birney E, Stamatoyannopoulos JA, Dutta A, Guigó R, Gingeras TR, et al. Identification and analysis of functional elements in $1 \%$ of the human genome by the ENCODE pilot project. Nature 2007;447:799-816.

3. ENCODE Project Consortium, Myers RM, Stamatoyannopoulos J, Snyder M, Dunham I, Hardison RC, et al. A user's guide to the encyclopedia of DNA elements (ENCODE). PLoS Biol 2011;9:e1001046.

4. ENCODE Project Consortium, Dunham I, Kundaje A, Aldred SF, Collins PJ, Davis CA, et al. An integrated encyclopedia of DNA elements in the human genome. Nature 2012;489:57-74.

5. Strahl BD, Allis CD. The language of covalent histone modifications. Nature 2000;403:41-45.

6. Heintzman ND, Hon GC, Hawkins RD, Kheradpour P, Stark
A, Harp LF, et al. Histone modifications at human enhancers reflect global cell-type-specific gene expression. Nature 2009; 459:108-112.

7. Andersson R, Enroth S, Rada-Iglesias A, Wadelius C, Komorowski J. Nucleosomes are well positioned in exons and carry characteristic histone modifications. Genome Res 2009; 19:1732-1741.

8. Luco RF, Pan Q, Tominaga K, Blencowe BJ, Pereira-Smith OM, Misteli T. Regulation of alternative splicing by histone modifications. Science 2010;327:996-1000.

9. Kouzarides T. Chromatin modifications and their function. Cell 2007;128:693-705.

10. Zhang ZD, Paccanaro A, Fu Y, Weissman S, Weng Z, Chang J, et al. Statistical analysis of the genomic distribution and correlation of regulatory elements in the ENCODE regions. Genome Res 2007;17:787-797.

11. Johnson DS, Li W, Gordon DB, Bhattacharjee A, Curry B, Ghosh J, et al. Systematic evaluation of variability in ChIP-chip experiments using predefined DNA targets. Genome Res 2008;18:393-403.

12. Sboner A, Mu XJ, Greenbaum D, Auerbach RK, Gerstein MB. The real cost of sequencing: higher than you think! Genome Biol 2011;12:125.

13. Ho JW, Bishop E, Karchenko PV, Nègre N, White KP, Park PJ. ChIP-chip versus ChIP-seq: lessons for experimental design and data analysis. BMC Genomics 2011;12:134.

14. Park PJ. ChIP-seq: advantages and challenges of a maturing technology. Nat Rev Genet 2009;10:669-680.

15. Landt SG, Marinov GK, Kundaje A, Kheradpour P, Pauli F, Batzoglou S, et al. ChIP-seq guidelines and practices of the ENCODE and modENCODE consortia. Genome Res 2012;22: 1813-1831.

16. Chen Y, Negre N, Li Q, Mieczkowska JO, Slattery M, Liu T, et al. Systematic evaluation of factors influencing ChIP-seq fidelity. Nat Methods 2012;9:609-614.

17. Egelhofer TA, Minoda A, Klugman S, Lee K, KolasinskaZwierz P, Alekseyenko AA, et al. An assessment of histone-modification antibody quality. Nat Struct Mol Biol 2011; 18:91-93.

18. Djebali S, Davis CA, Merkel A, Dobin A, Lassmann T, Mortazavi A, et al. Landscape of transcription in human cells. Nature 2012;489:101-108.

19. Flicek P, Amode MR, Barrell D, Beal K, Brent S, Carvalho-Silva D, et al. Ensembl 2012. Nucleic Acids Res 2012;40:D84-D90.

20. Guttman M, Amit I, Garber M, French C, Lin MF, Feldser D, et al. Chromatin signature reveals over a thousand highly conserved large non-coding RNAs in mammals. Nature 2009;458: 223-227.

21. Guttman M, Garber M, Levin JZ, Donaghey J, Robinson J, Adiconis $\mathrm{X}$, et al. Ab initio reconstruction of cell type-specific transcriptomes in mouse reveals the conserved multi-exonic structure of lincRNAs. Nat Biotechnol 2010;28:503-510.

22. Mercer TR, Dinger ME, Mattick JS. Long non-coding RNAs: insights into functions. Nat Rev Genet 2009;10:155-159.

23. Katayama S, Tomaru Y, Kasukawa T, Waki K, Nakanishi M, Nakamura $\mathrm{M}$, et al. Antisense transcription in the mammalian transcriptome. Science 2005;309:1564-1566. 
24. Derrien T, Johnson R, Bussotti G, Tanzer A, Djebali S, Tilgner $\mathrm{H}$, et al. The GENCODE $\mathrm{v}$ catalog of human long noncoding RNAs: analysis of their gene structure, evolution, and expression. Genome Res 2012;22:1775-1789.

25. Reed R. Coupling transcription, splicing and mRNA export. Curr Opin Cell Biol 2003;15:326-331.

26. Kornblihtt AR, de la Mata M, Fededa JP, Munoz MJ, Nogues G. Multiple links between transcription and splicing. RNA 2004; 10:1489-1498.

27. Listerman I, Sapra AK, Neugebauer KM. Cotranscriptional coupling of splicing factor recruitment and precursor messenger RNA splicing in mammalian cells. Nat Struct Mol Biol 2006;13:815-822.

28. Vargas DY, Shah K, Batish M, Levandoski M, Sinha S, Marras $\mathrm{SA}$, et al. Single-molecule imaging of transcriptionally coupled and uncoupled splicing. Cell 2011;147:1054-1065.

29. Tilgner H, Knowles DG, Johnson R, Davis CA, Chakrabortty S, Djebali $S$, et al. Deep sequencing of subcellular RNA fractions shows splicing to be predominantly co-transcriptional in the human genome but inefficient for lncRNAs. Genome Res 2012;22:1616-1625

30. Hon G, Wang W, Ren B. Discovery and annotation of functional chromatin signatures in the human genome. PLoS Comput Biol 2009;5:e1000566.

31. Kolasinska-Zwierz P, Down T, Latorre I, Liu T, Liu XS, Ahringer J. Differential chromatin marking of introns and expressed exons by H3K36me3. Nat Genet 2009;41:376-381.

32. Nahkuri S, Taft RJ, Mattick JS. Nucleosomes are preferentially positioned at exons in somatic and sperm cells. Cell Cycle 2009;8:3420-3424.

33. Spies N, Nielsen CB, Padgett RA, Burge CB. Biased chromatin signatures around polyadenylation sites and exons. Mol Cell 2009;36:245-254.

34. Tilgner H, Nikolaou C, Althammer S, Sammeth M, Beato M, Valcárcel J, et al. Nucleosome positioning as a determinant of exon recognition. Nat Struct Mol Biol 2009;16:996-1001.

35. Li M, Wang IX, Li Y, Bruzel A, Richards AL, Toung JM, et al. Widespread RNA and DNA sequence differences in the human transcriptome. Science 2011;333:53-58.

36. Pickrell JK, Gilad Y, Pritchard JK. Comment on "Widespread RNA and DNA sequence differences in the human transcriptome". Science 2012;335:1302.

37. Kleinman CL, Majewski J. Comment on "Widespread RNA and DNA sequence differences in the human transcriptome". Science 2012;335:1302.

38. Lin W, Piskol R, Tan MH, Li JB. Comment on "Widespread RNA and DNA sequence differences in the human transcriptome". Science 2012;335:1302.

39. Park E, Williams B, Wold BJ, Mortazavi A. RNA editing in the human ENCODE RNA-seq data. Genome Res 2012;22: 1626-1633.

40. Peng Z, Cheng Y, Tan BC, Kang L, Tian Z, Zhu Y, et al. Comprehensive analysis of RNA-Seq data reveals extensive RNA editing in a human transcriptome. Nat Biotechnol 2012; 30:253-260.

41. Mighell AJ, Smith NR, Robinson PA, Markham AF. Vertebrate pseudogenes. FEBS Lett 2000;468:109-114.
42. Harrison PM, Echols N, Gerstein MB. Digging for dead genes: an analysis of the characteristics of the pseudogene population in the Caenorhabditis elegans genome. Nucleic Acids Res 2001;29:818-830.

43. Echols N, Harrison P, Balasubramanian S, Luscombe NM, Bertone $\mathrm{P}$, Zhang Z, et al. Comprehensive analysis of amino acid and nucleotide composition in eukaryotic genomes, comparing genes and pseudogenes. Nucleic Acids Res 2002;30: 2515-2523.

44. Balakirev ES, Ayala FJ. Pseudogenes: are they "junk" or functional DNA? Annu Rev Genet 2003;37:123-151.

45. Poliseno L, Salmena L, Zhang J, Carver B, Haveman WJ, Pandolfi PP. A coding-independent function of gene and pseudogene mRNAs regulates tumour biology. Nature 2010;465: 1033-1038.

46. Tam OH, Aravin AA, Stein P, Girard A, Murchison EP, Cheloufi $\mathrm{S}$, et al. Pseudogene-derived small interfering RNAs regulate gene expression in mouse oocytes. Nature 2008;453:534-538.

47. Watanabe T, Totoki Y, Toyoda A, Kaneda M, KuramochiMiyagawa S, Obata Y, et al. Endogenous siRNAs from naturally formed dsRNAs regulate transcripts in mouse oocytes. Nature 2008;453:539-543.

48. Sasidharan R, Gerstein M. Genomics: protein fossils live on as RNA. Nature 2008;453:729-731.

49. Guo X, Zhang Z, Gerstein MB, Zheng D. Small RNAs originated from pseudogenes: cis- or trans-acting? PLoS Comput Biol 2009;5:e1000449.

50. Pei B, Sisu C, Frankish A, Howald C, Habegger L, Mu XJ, et al. The GENCODE pseudogene resource. Genome Biol 2012;13: R51.

51. Kim TK, Hemberg M, Gray JM, Costa AM, Bear DM, Wu J, et al. Widespread transcription at neuronal activity-regulated enhancers. Nature 2010;465:182-187.

52. Core LJ, Waterfall JJ, Lis JT. Nascent RNA sequencing reveals widespread pausing and divergent initiation at human promoters. Science 2008;322:1845-1848.

53. Wang D, Garcia-Bassets I, Benner C, Li W, Su X, Zhou Y, et al. Reprogramming transcription by distinct classes of enhancers functionally defined by eRNA. Nature 2011;474:390-394.

54. Kodzius R, Kojima M, Nishiyori H, Nakamura M, Fukuda S, Tagami M, et al. CAGE: cap analysis of gene expression. Nat Methods 2006;3:211-222.

55. Ng P, Wei CL, Sung WK, Chiu KP, Lipovich L, Ang CC, et al. Gene identification signature (GIS) analysis for transcriptome characterization and genome annotation. Nat Methods 2005;2: 105-111.

56. Wang Z, Gerstein M, Snyder M. RNA-Seq: a revolutionary tool for transcriptomics. Nat Rev Genet 2009;10:57-63.

57. Dong X, Greven MC, Kundaje A, Djebali S, Brown JB, Cheng $\mathrm{C}$, et al. Modeling gene expression using chromatin features in various cellular contexts. Genome Biol 2012;13:R53.

58. Karlic R, Chung HR, Lasserre J, Vlahovicek K, Vingron M. Histone modification levels are predictive for gene expression. Proc Natl Acad Sci U S A 2010;107:2926-2931.

59. Costa IG, Roider HG, do Rego TG, de Carvalho Fde A. Predicting gene expression in $\mathrm{T}$ cell differentiation from histone modifications and transcription factor binding affinities 
by linear mixture models. BMC Bioinformatics 2011;12 Suppl 1:S29.

60. Fawcett T. An introduction to ROC analysis. Pattern Recognit Lett 2006;27:861-874.

61. Natarajan A, Yardimci GG, Sheffield NC, Crawford GE, Ohler U. Predicting cell-type-specific gene expression from regions of open chromatin. Genome Res 2012;22:1711-1722.

62. Hoffman MM, Ernst J, Wilder SP, Kundaje A, Harris RS, Libbrecht $\mathrm{M}$, et al. Integrative annotation of chromatin elements from ENCODE data. Nucleic Acids Res 2013;41:827-841.

63. Ernst J, Kheradpour P, Mikkelsen TS, Shoresh N, Ward LD, Epstein CB, et al. Mapping and analysis of chromatin state dynamics in nine human cell types. Nature 2011;473:43-49.

64. Won, KJ, Zhang X, Wang T, Ding B, Raha D, Snyder M, et al. Comparative annotation of functional regions in the human genome using epigenome data. Nucleic Acids Rese 2013;41:
4423-4432.

65. Shin H, Liu T, Manrai AK, Liu XS. CEAS: cis-regulatory element annotation system. Bioinformatics 2009;25:2605-2606.

66. Ye T, Krebs AR, Choukrallah MA, Keime C, Plewniak F, Davidson I, et al. seqMINER: an integrated ChIP-seq data interpretation platform. Nucleic Acids Res 2011;39:e35.

67. Gerstein MB, Kundaje A, Hariharan M, Landt SG, Yan KK, Cheng C, et al. Architecture of the human regulatory network derived from ENCODE data. Nature 2012;489:91-100.

68. Neph S, Vierstra J, Stergachis AB, Reynolds AP, Haugen E, Vernot $\mathrm{B}$, et al. An expansive human regulatory lexicon encoded in transcription factor footprints. Nature 2012;489: 83-90.

69. Sanyal A, Lajoie BR, Jain G, Dekker J. The long-range interaction landscape of gene promoters. Nature 2012;489: 109-113. 